\title{
Coordinated Operation of Autonomous Underwater and Surface Vehicles
}

\author{
Aníbal Matos and Nuno Cruz \\ Faculdade de Engenharia da Universidade do Porto \\ Instituto de Sistemas e Robótica - Porto \\ Rua Dr. Roberto Frias, 4200-465 Porto \\ Portugal \\ \{anibal,nacruz $\} @$ fe.up.pt
}

\begin{abstract}
This work addresses the coordinated operation of an autonomous underwater vehicle and an autonomous surface vessel and its main goal is the development of an infrastructure that allows the surface vessel to dynamically position itself above the underwater vehicle while the later one is collecting data and navigating in long baseline mode using a set of beacons installed in the operation area. Besides a formal statement of the coordination problem, we present results both from real experiments and from simulations that illustrate the proposed solution.
\end{abstract}

\section{INTRODUCTION}

This paper addresses the guidance and control of an autonomous surface vehicle (ASV) to perform coordinated operations with an autonomous underwater vehicle (AUV). The main goal is to position the ASV directly above the AUV while this one is collecting data and navigating in long baseline mode. The ASV determines the realtime position of the AUV by tapping the acoustic channel and listening to the acoustic signals exchanged between the AUV and the acoustic beacons deployed in the operation area.

The paper starts with a brief description of the vehicles addressed in this work. Then, section III presents the control system of the ASV and section IV describes the long baseline navigation system of the AUV as well as the tracking mechanism that allows for the real time estimation of the AUV position by the ASV. Section V describes how the trajectory of the AUV is estimated and section VI presents the guidance algorithm used by the ASV, while positioning itself above the AUV.

\section{EXPERIMENTAL SETUP}

The vehicles used in this work are the OS8 AUV and the Zarco ASV, both developed at Porto University. OS8 (fig. 1) is a 8-inch diameter vehicle, with a total length of 1.5 $\mathrm{m}$, and weighting about $40 \mathrm{~kg}$. It possesses a plastic hull, with a dry mid body (for electronics and bats) and additional rings to accommodate sensors and actuators. It is propelled by two horizontal thrusters, located at the rear, and two vertical thrusters, one at the front and the other at the rear. This configuration allows for small operational speeds and high maneuverability, including pure vertical motions. It is equipped with an omnidirectional acoustic transducer and with an electronic system that allows for long baseline navigation.

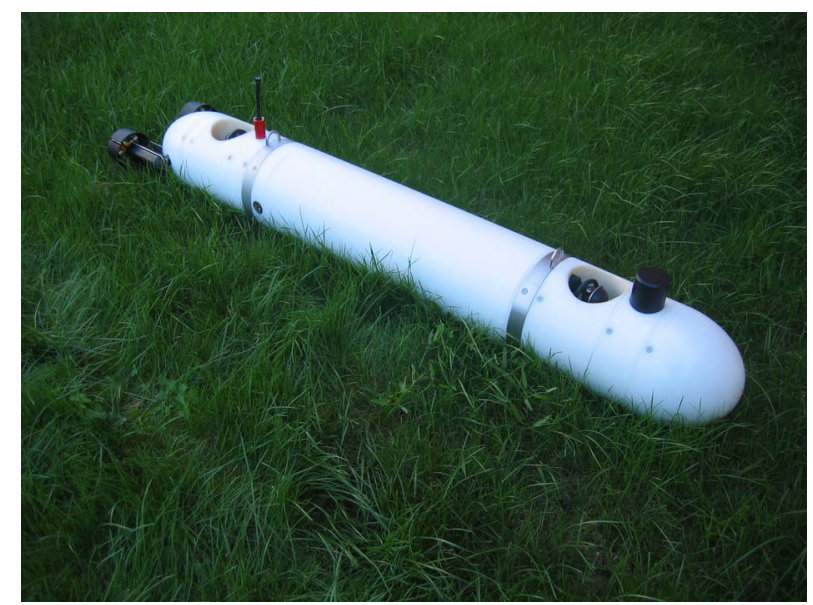

Fig. 1. OS8 AUV.

Its main applications include oceanographic and environmental monitoring, as well as autonomous inspection operations.

Zarco (fig. 2) is a small size craft designed to perform autonomous missions mainly in rivers dams and estuarine environments, either serving as a moving navigation beacon for underwater vehicles or collecting high resolution interferometric synthetic aperture sonar data.

The vehicle is based on a catamaran type floating structure with 1.5 meters long and an overall width about 1 meter. In its basic configuration, the vehicle weights a total of 45 $\mathrm{kg}$, and has an additional payload capacity of more than 25 $\mathrm{kg}$. The vehicle is powered by two electric motors capable of delivering a total thrust of $250 \mathrm{~N}$, which allows for a maximum speed exceeding 3 knots. A set of easy replaceable rechargeable lead acid batteries provide energy for more than 4 hours of continuous operation. The vehicle also carries an onboard computer responsible for the execution of autonomous or remotely controlled missions and for the storage of collected payload data. The navigation system relies on data provided by a magnetic compass, an inertial measurement unit, and a $\mathrm{L} 1+\mathrm{L} 2$ RTK pair of GPS receivers, and is able to estimate in real time the position and attitude of the vehicle with high accuracy. A WiFi link connects the boat to a shore station, 


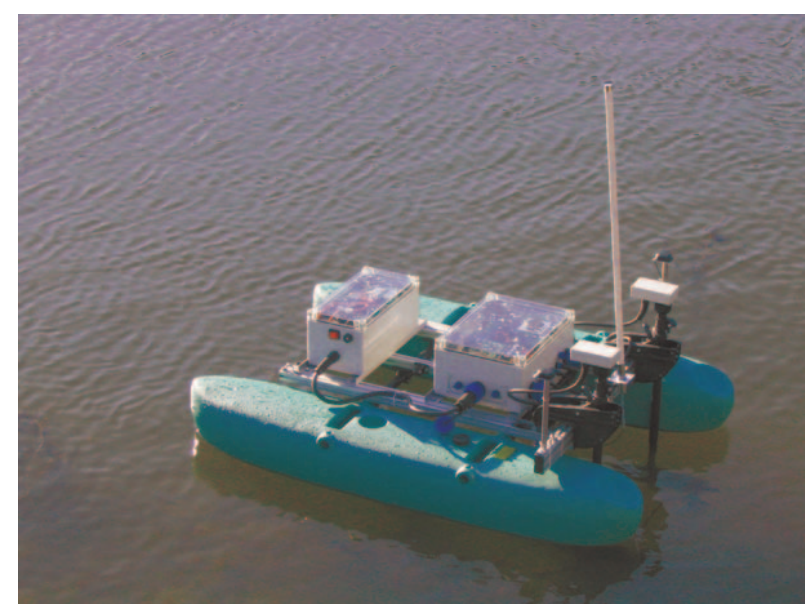

Fig. 2. Zarco ASV.

allowing for the transmission of GPS base station data and for the supervision of the vehicle autonomous operations. In order to allow for different configurations, either in terms of floatation, propulsion, on board energy, or payload sensors, the whole system was developed in a modular fashion, mainly using off-the-shelf components.

\section{ASV CONTROL}

For the purpose of the surface vessel maneuvering it is usual to consider a 3 DOF model. This model can be derived from a general 6 DOF model of a partially submerged body, considering that ([1]):

- the vehicle has $x z$-plane symmetry $\left(I_{x y}=I_{y z}=0\right)$, and

- the heave, roll and pitch modes can be ignored $(w=p=$ $q=\dot{w}=\dot{p}=\dot{q}=0$ ).

In this case, the motion of the ASV is governed by the equations

$$
\begin{aligned}
& m\left(\dot{u}-v r-y_{G} \dot{r}-x_{G} r^{2}\right)=X \\
& m\left(\dot{v}-u r+x_{G} \dot{r}-y_{G} r^{2}\right)=Y \\
& I_{z} \dot{r}+m\left[x_{G}(\dot{v}+u r)-y_{G}(\dot{u}-v r)=N\right.
\end{aligned}
$$

where $X, Y$ and $N$ represent the external forces and moments acting on the vehicle.

Considering that the body fixed frame is located at the centre of mass of the vehicle $\left(x_{G}=y_{G}=0\right)$, these equations can be written as

$$
\begin{aligned}
& m(\dot{u}-v r)=X \\
& m(\dot{v}-u r)=Y \\
& I_{z} \dot{r}=N
\end{aligned}
$$

Neglecting the wind effects and considering that the horizontal components of the water current are constant, $u$ and $v$ can be taken as the components of the vehicle velocity with respect to the water. In this case, the external forces $X$ and $Y$ only depend on the velocity of the vehicle with respect to the water and on the vehicle actuation. Furthermore, the evolution of position of the vehicle in a earth fixed frame and of its heading with respect to such frame are given by

$$
\begin{aligned}
& \dot{x}=u \cos \psi-v \sin \psi+c_{x} \\
& \dot{y}=u \sin \psi+v \cos \psi+c_{y} \\
& \dot{\psi}=r
\end{aligned}
$$

where $c_{x}$ and $c_{y}$ are the $x$ and $y$ components of the water current.

Due to the symmetry of the vehicle, we will further assume that $X, Y$ and $N$ are just given by

$$
\begin{aligned}
& X=X_{\dot{u}} \dot{u}+X_{u} u+X_{u|u|} u|u|+X_{a c t} \\
& Y=Y_{\dot{v}} \dot{v}+Y_{v} v+Y_{v|v|} v|v| \\
& N=N_{\dot{r}} \dot{r}+N_{r} r+N_{r|r|} r|r|+N_{a c t}
\end{aligned}
$$

\section{A. Vehicle actuation}

The vehicle is propelled by two forward thrusters. The port side thruster is located at $(-b,-l)$, while the starboard side one is located at $(+b,-l)$, both in CM coordinates.

Consider that the force provided by the port thruster is $F_{1}$, while the force provided by the starboard thruster is $F_{2}$. When the thrusters are in perfect alignment conditions, the net actuation of the vehicle is given by

$$
\begin{aligned}
& X_{a c t}=F_{1}+F_{2} \\
& Y_{a c t}=0 \\
& N_{a c t}=b \cdot\left(F_{1}-F_{2}\right)
\end{aligned}
$$

However, there are always small deviations in the thrusters positions and alignments which will affect the overall actuation of the vehicle. In practice, the deviation in the thrusters positions will be negligible, due to their rigid attachment to the vehicle frame. On the other hand, thruster alignment is adjusted when launching the vehicle, so minor differences might appear from operation to operation. Let us consider now that, instead of being perfectly align along the vehicle principal axis, the port and starboard thrusters are aligned along directions $\phi_{1}$ and $\phi_{2}$, respectively, where $\phi_{1}$ and $\phi_{2}$ are small. In such case, the net vehicle actuation is

$$
\begin{aligned}
& X_{a c t}=F_{1}+F_{2} \\
& Y_{a c t}=F_{1} \cdot \phi_{1}+F_{2} \cdot \phi_{2} \\
& N_{a c t}=b \cdot\left(F_{1}-F_{2}\right)-l \cdot\left(F_{1} \cdot \phi_{1}+F_{2} \cdot \phi_{2}\right)
\end{aligned}
$$

where we can note that the misalignments result in the appearance of a lateral force and in the modification of the net torque.

Another issue that must be considered is the thrusters dynamics. Although a complete model takes into account the internal electro-mechanical dynamics as well as the propellerwater interaction, we will disregard such dynamics since they are sufficiently faster than the dynamics associated to the vehicle motion. Thus, we will consider for each thruster a static relationship $F=f(U)$, where $F$ is the force provided by the thruster, and $U$ the actuation command, in a normalized -100 to +100 scale. 
Ideally, both thrusters would be characterized by the same function, but usually it is not possible to have thrusters with equal characteristics. To account for such differences we will consider that the input output relationships for the port and starboard thrusters are $f_{1}=f+\Delta f$ and $f_{2}=f-\Delta f$, respectively, where $\Delta f$ is the first order mismatch between the thrusters, resulting then that $F_{1}=f_{1}\left(U_{1}\right)$ and $F_{2}=f_{2}\left(U_{2}\right)$.

To simplify the control of the vehicle it is usual to decouple the longitudinal and the rotational motions. The longitudinal net actuation $X_{a c t}$ is used to control the vehicle speed, while the rotational actuation $N_{a c t}$ is used to guide the vehicle along a given path. Separate controllers are then used to adjust, in real time, the actuation $X_{a c t}$ and $N_{a c t}$. From these, the port and starboard thruster forces are then computed according to

$$
\begin{aligned}
& F_{1}=\frac{X_{a c t}}{2}+\frac{N_{a c t}}{b} \\
& F_{2}=\frac{X_{a c t}}{2}-\frac{N_{a c t}}{b},
\end{aligned}
$$

and, finally, the normalized actuation is then obtained from

$$
\begin{aligned}
U_{1} & =f_{1}^{-1}\left(F_{1}\right) \\
U_{2} & =f_{2}^{-1}\left(F_{2}\right) .
\end{aligned}
$$

\section{B. Line tracking}

We will consider that the vehicle is intended to move along rectilinear trajectories in the horizontal plane at constant speeds. To derive a controller for this motion, we can take into account just the vehicle steering dynamics

$$
\begin{aligned}
& m\left(\dot{v}-u_{0} r\right)=Y \\
& I_{z} \dot{r}=N
\end{aligned}
$$

where $u_{0}$ is the intended forward speed. For the purpose of line tracking, it is reasonable to assume that $v$ and $r$ will be small and, therefore, the second order terms in $Y$ and $N$ and be disregarded, resulting

$$
\begin{aligned}
& Y=Y_{\dot{v}} \dot{v}+Y_{v} v \\
& N=N_{\dot{r}} \dot{r}+N_{r} r+N_{a c t}
\end{aligned}
$$

When driving the vehicle towards a given line, the most important quantities to take into account are the off-track error $d$, and the heading error $\beta$ (see figure 3), as well as the crosstrack water current $c_{d}$.

To simplify the derivation of the control law we will consider the tracking of the $x$ axis line. In this case we have

$$
\begin{aligned}
& d=y \\
& \beta=-\psi \\
& c_{d}=c_{y}
\end{aligned}
$$

The steering dynamics will then be

$$
\begin{aligned}
& \left(m-Y_{\dot{v}}\right) \dot{v}=Y_{v} v+m u_{0} r \\
& \left(I_{z}-N_{\dot{r}}\right) \dot{r}=N_{r} r+N_{a c t} \\
& \dot{y}=u_{0} \sin \psi+v \cos \psi+c_{y} \\
& \dot{\psi}=r
\end{aligned}
$$

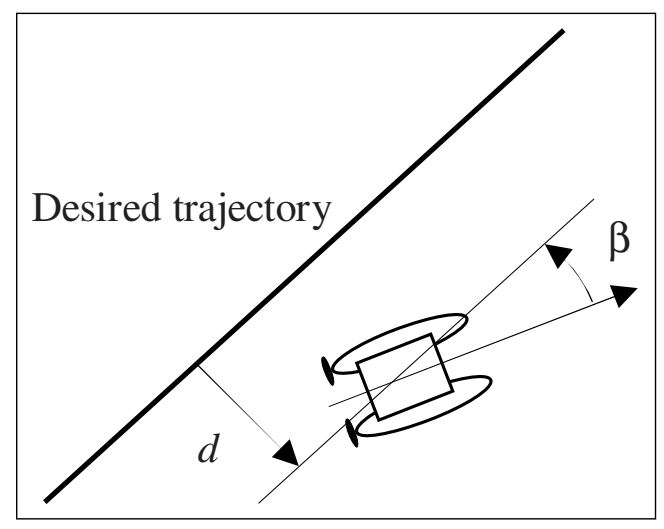

Fig. 3. Off-track $(d)$ and heading $(\beta)$ errors.

and its steady state solution is naturally given by $v=r=0$ and $\psi=\psi_{0}=-\arcsin \frac{c_{y}}{u_{0}}$.

Since, in general the water current $c_{d}=c_{y}$ is unknown (and difficult to estimate directly), to ensure that steady state tracking error is null it is necessary to consider an additional state $l=\int y d t$.

Linearizing the augmented dynamics around the equilibrium point conducts to the following equations

$$
\begin{aligned}
& \dot{v}=\frac{Y_{v}}{m-Y_{\dot{v}}} v+\frac{m u_{0}}{m-Y_{\dot{v}}} r \\
& \dot{r}=\frac{N_{r}}{I_{z}-N_{\dot{r}}} r+\frac{1}{I_{z}-N_{\dot{r}}} N_{a c t} \\
& \dot{y}=\cos \psi_{0} v+u_{0} \cos \psi_{0} \psi \\
& \dot{\psi}=r \\
& \dot{l}=y .
\end{aligned}
$$

The line tracking control system considered here is constituted by a two stage controller defined by

$$
\begin{aligned}
& \psi_{r e f}=\psi_{t r a j}-k_{y} y-k_{l} l \\
& N_{a c t}=k_{\psi}\left(\psi_{r e f}-\psi\right)-k_{r} r
\end{aligned}
$$

where the first law is a guidance controller that computes the desired heading reference, while the second one is a simple PD feedback law; $\psi_{t r a j}$ is just the heading of the trajectory that the vehicle must track.

In can be shown that when $u_{0} \neq 0$ and $\left|c_{y}\right| \leq u_{0}$, the linearized steering system (1) is completely controllable. In that case, it is possible to determine feedback gains $k_{y}, k_{l}, k_{\psi}$ and $k_{r}$ such that the closed loop system is stable.

A final note should be said about the implementation of the feedback controller. Due to saturation of the inputs, namely $N_{a c t}$, integral wind-up effects might arise. To overcome such difficulties, the heading reference is in fact given by

$$
\psi_{\text {ref }}=\psi_{t r a j}-\arctan \left(k_{y} y+k_{l} l\right) .
$$

\section{Vehicle performance}

Figure 4 shows the evolution of Zarco while autonomously describing a $100 \mathrm{~m} \times 50 \mathrm{~m}$ rectangle at velocities ranging from 0.5 to $1.5 \mathrm{~m} / \mathrm{s}$, in the counterclockwise direction. 


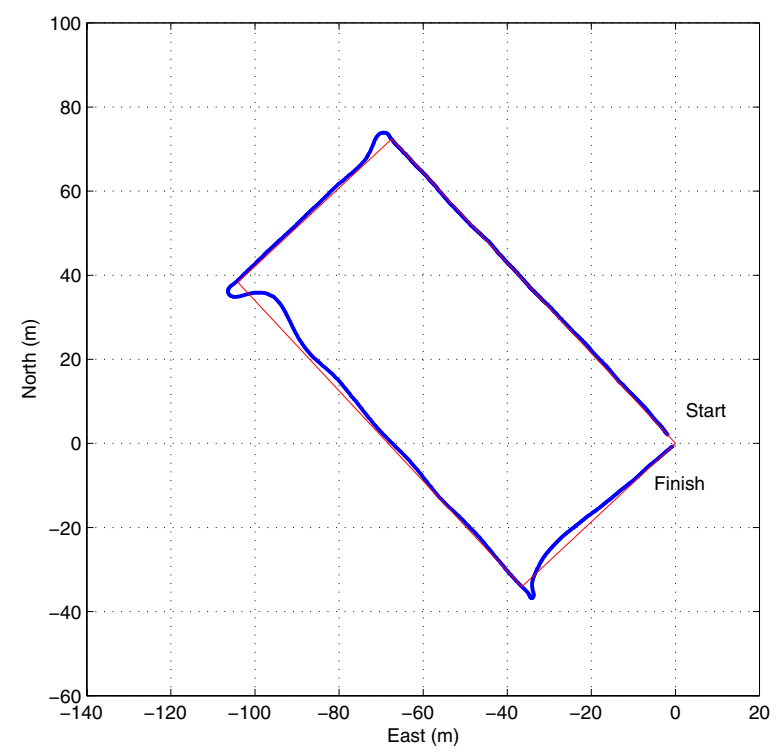

Fig. 4. Zarco sample mission.

The overshoots exhibited at the end of each line are due the fact that the vehicle only starts tracking a new line after crossing the end of the previous one.

The performance of the control system can be further assessed considering the off-track error when following straight lines. Figure 5 shows this error for the first $100 \mathrm{~m}$ line. Typically, as can be seen in this figure, the error is almost always below $0.5 \mathrm{~m}$, and its root mean square value is in the order of $0.2 \mathrm{~m}$.

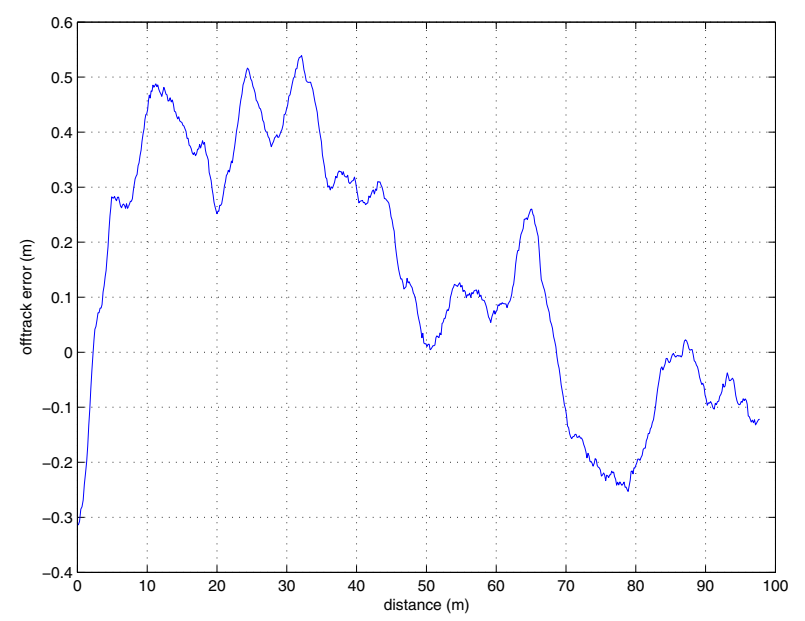

Fig. 5. Line tracking performance.

\section{AUV NAVIGATION AND TRACKING}

To determine its position in the horizontal plane, the AUV relies on a long baseline acoustic network. The network is composed by a set of acoustic beacons that are deployed in the operation area. While executing its mission, the vehicle

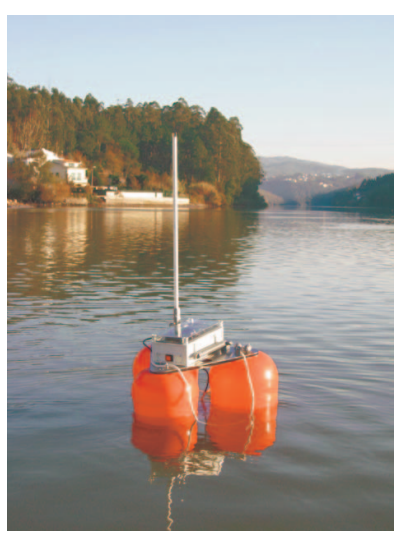

Fig. 6. Buoy connected to acoustic beacon.

interrogates the beacons to determine its distance to each of them. Using at least two beacons, located in different places, it is them possible to determine the position of the AUV. This positioning method has a slow update rate (usually more than 1 second for each range measurement) and therefore these range measurements have to be fused together with dead reckoning data to provide the control system of the AUV with good enough position estimates of the vehicle. This data fusion is performed by a Kalman filter ([2]) based algorithm, as the one described in [3].

While navigating, the vehicle onboard software completely controls the exchange of acoustic signals and obtains the range measurements one at a time. To determine its range to a given beacon, the vehicle first sends an interrogation signal. All the beacons detect that signal but only the one identified with such interrogation signal will reply with a signal of another predefined frequency. Upon detection of the beacon reply, the vehicle measures the elapse time, computes the range, and interrogates another beacon, starting a new range measurement.

The external tracking of the AUV can be made just by listening to the acoustic signals exchanged between the vehicle and the acoustic beacons and relies on the algorithm proposed in [4]. To make it possible, the AUV navigation system has just to send each interrogation signal a deterministic amount of time immediately after having received a beacon reply.

The exchanged signals are reported to a shore station and to the ASV by a radio linked surface buoy which is connected to one of the acoustic beacons (fig. 6).

\section{AUV TRAJECTORY ESTIMATION}

Even though the external tracking estimates of the vehicle position can be properly filtered, as described in [5], their update rate is quite slow (typically more than 2 seconds for a completely new position estimate) and their residual error can be as high as 10 meters, especially when the AUV is turning. These characteristics makes such estimates unsuited for direct use as inputs to the ASV control system.

On the other hand, during a typical mission, the AUV will try to describe a sequence of straight lines, according to an $a$ 
priori mission plan, or defined on-the-fly in adaptive sampling missions [6].

Since the trajectory tracking error of the AUV is much smaller that the external tracking position estimate it is advantageous to use such knowledge about the AUV evolution to produce a more adequate input to the ASV control system.

In this way, we will consider that the AUV will evolve along a sequence of straight lines, each one with a constant velocity. For each line, the evolution of the vehicle is given by

$$
\begin{aligned}
& x(t)=x_{0}+v_{x} t \\
& y(t)=y_{0}+v_{y} t
\end{aligned}
$$

where $\left(x_{0}, y_{0}\right)$ is its horizontal position at $t=0$ and $\left(v_{x}, v_{y}\right)$ is its horizontal velocity.

Since there is no communication with the AUV, it is not simple to understand when the AUV finished moving along a given line and started the next one. Moreover, the new line can be defined on-the-fly. It is then impossible to a priori partition the mission time in predefined number of intervals, each one with a known duration, and estimate $x_{0}, y_{0}, v_{x}, v_{y}$ for each time interval.

Since equations (2) and (3) exhibit a linear dependence of the vehicle position on the trajectory defining parameters, we will use a recursive least squares with forgetting algorithm ([7] and [8]) to estimate in real time the parameters that describe the AUV trajectory.

From a sequence of estimated positions of the AUV and their corresponding time stamps, $(x, y, t)_{i}$, the least squares algorithm produces a sequence of estimated trajectory parameters $\left(\hat{x}_{0}, \hat{y}_{0}, \hat{v}_{x}, \hat{v}_{y}\right)_{i}$.

Figures (7) and (8) show, respectively, the real time estimation of the vehicle velocity $\left(v_{x}, v_{y}\right)$ and its course

$$
\psi_{A U V}=\arctan \frac{v_{y}}{v_{x}}
$$

when the AUV is making a 90 degrees turn between two straight lines.

\section{ASV GUIDANCE}

The coordination of the AUV and ASV motions is assured by using the estimated trajectory of the AUV as the guiding data of the ASV control system.

The estimated trajectory parameters $x_{0}, y_{0}, v_{x}$ and $v_{y}$ are used, in a first stage, to obtain $\psi_{A U V}$, defined above, $v_{A U V}=$ $\sqrt{v_{x}^{2}+v_{y}^{2}}$, and $h_{A U V}$, defined by

$$
h_{A U V}=\frac{v_{y} x_{0}-v_{x} y_{0}}{v_{A U V}},
$$

which is the offset from the origin of the current AUV trajectory. At a given time instant $t$, the position of the AUV projected on the estimated trajectory is then given by

$$
s_{A U V}=\frac{x_{0} v_{x}+y_{0} v_{y}}{v_{A U V}}+v_{A U V} t
$$

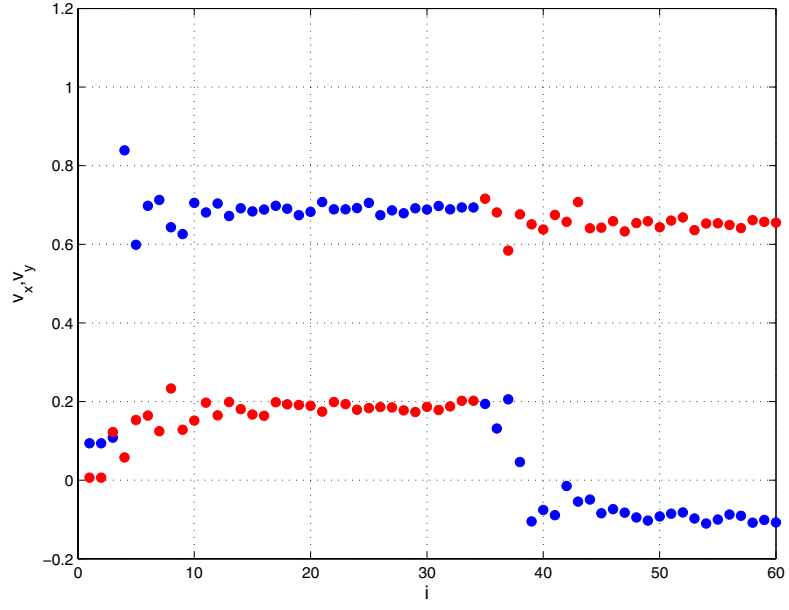

Fig. 7. Estimated velocity of the AUV ( $v_{x}$ in blue and $v_{y}$ in red).

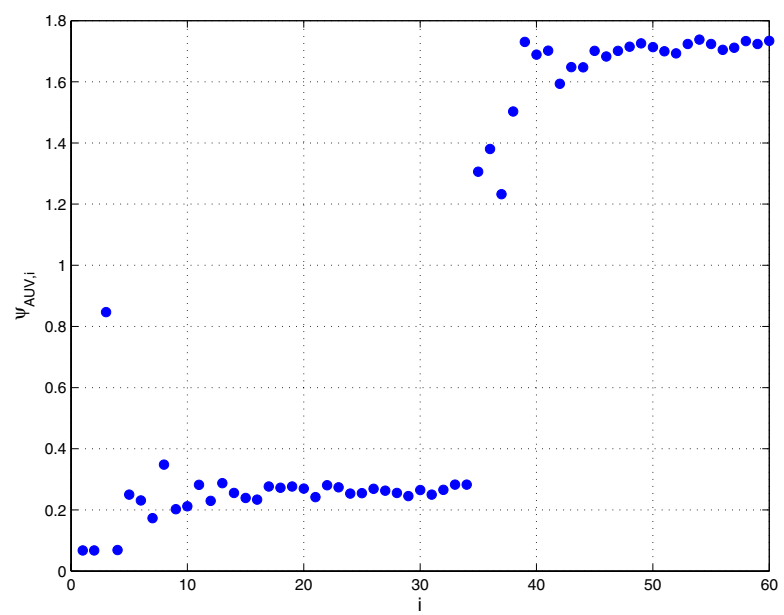

Fig. 8. Estimated course of the AUV.

Since the ASV control is decoupled in longitudinal and rotational modes, two separate inputs must be defined. For the longitudinal mode, the actuation force $X_{a c t}$ is defined by

$$
\begin{aligned}
X_{a c t}= & F_{f}\left(v_{A U V}\right)+k_{v}\left(v_{A U V}-v\right)+k_{s}\left(s_{A U V}-s\right)+ \\
& +k_{i} \int\left(s_{A U V}-s\right) d t
\end{aligned}
$$

where $F_{f}(\cdot)$ is a feedforward compensation term that roughly accounts for the force required to maintain a given velocity, $v$ is the ASV velocity, and $s$ is the projected position of the ASV in the AUV trajectory, given by

$$
s=\frac{x v_{x}+y v_{y}}{v_{A U V}},
$$

where $(x, y)$ is the current position of the ASV.

The rotational torque $N_{a c t}$ is defined by

$N_{a c t}=k_{\psi}\left(\psi_{A U V}-\arctan \left(k_{y} d+k_{l} \int d d t\right)-\psi\right)-k_{r} r$ 


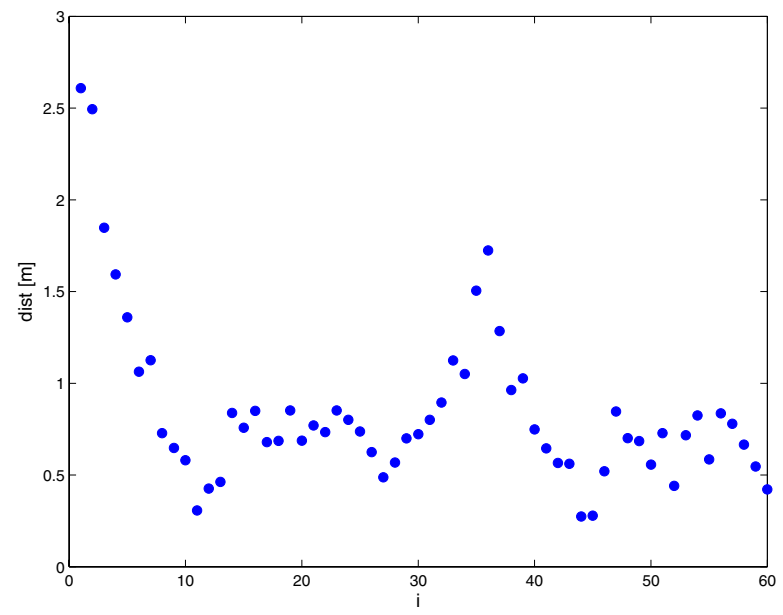

Fig. 9. Horizontal distance between AUV and ASV.

where $\psi$ is the ASV heading, $r$ is its heading rate, and $d$ is the cross track error, now given by

$$
d=\frac{v_{x} y+v_{y} x}{v_{A U V}}+h_{A U V} .
$$

Figure (9) presents the results of a simulation of the above guidance algorithm in terms of the distance between the two vehicles. This simulation was performed with real data of the AUV external trajectory estimation, corresponding to the 90 degrees turn presented before.

\section{ACKNOWLEDGMENT}

This work was supported by the project MUV - Multiple Underwater Vehicles - funded by FCT under Programa POSI (ref. POSI/SRI/47351/2002) and by the project INCORP Improved Navigation with Cooperative Robotic Platforms funded by FCT under Programa POSC (ref. POSC/EEASRI/59963/2004).

\section{REFERENCES}

[1] Thor I. Fossen. Guidance and Control of Ocean Vehicles. John Wiley \& Sons Ltd., England, 1994.

[2] A. Gelb, J. Kasper Jr., R. Nash Jr., C. Price, and A. Sutherland Jr. Applied Optimal Estimation. The MIT Press, Cambridge, MA, USA, 1996.

[3] A. Matos, N. Cruz, A. Martins, and F. Pereira. Development and implementation of a low-cost LBL navigation systems for an AUV. In Proc. of the MTS/IEEE Conf. Oceans'99, Seattle, WA, USA, September 1999.

[4] N. Cruz, L. Madureira, A. Matos, and F. Pereira. A versatile acoustic beacon for navigation and remote tracking of multiple underwater vehicles. In Proc. of the MTS/IEEE Conf. Oceans'01, Honolulu, HI, USA, November 2001.

[5] A. Matos and N. Cruz. Algorithms for external tracking of an AUV. In Proceedings of the 5th IFAC Symposium on Intelligent Autonomous Vehicles IAV'2004, Lisbon, Portugal, July 2004.

[6] Nuno Cruz, Aníbal Matos, and João Sousa. Small size AUVs: Operation results and new mission concepts. In Proceedings of the 5th IFAC Symposium on Intelligent Autonomous Vehicles IAV'2004, Lisbon, Portugal, July 2004.

[7] T. Hagglund. Recursive estimation of slowly time varying parameters. In Proceedings of IFAC, pages 1143-1148, 1985.

[8] A. Vahidi, A. Stefanopoulou, and H. Peng. Recursive least squares with forgetting for online estimation of vehicle mass and road grade: Theory and experiments. Vehicle Systems Dynamics, (43):31-57, 2005. 